\title{
VALIDAÇÃO DE TÉCNICA ANALÍTICA EM CROMATOGRAFIA EM CAMADA DELGADA COMPARATIVA PARA IDENTIFICAÇÃO DE FÁRMACOS ANOREXÍGENOS SINTÉTICOS EM PRODUTOS FITOTERÁPICOS
}

\author{
Validation of analytic technique in comparative thin layer chomatography for identification of \\ synthetic anorexic drugs in natural medecins
}

\author{
Flaubertt S. de Azeredo'; Renato I. Guimarães'; José R. de Paula²; \\ Luiz C. da Cunha ${ }^{1}$ \\ 1. Núcleo de Estudos e Pesquisas Tóxico-farmacológicas (NEPET-UFG). \\ 2. Laboratório de Farmacognosia, - Faculdade de Farmácia / UFG. \\ Endereço eletrônico: Iccunha@farmacia.ufg.br, sítio eletrônico: www.farmacia.ufg.br .
} Apoio: SECTEC/CONCITEG; PIBIC/CNPq; AFAGO

Recebido em 05/11/2004 - Aceito em 23/11/2004

\begin{abstract}
RESUMO: Há relatos de fármacos sintéticos psicoativos, que possuem restrições de venda e uso, não mencionados na composição de fitoterápicos emagrecedores comercializados livremente. Sendo necessário identificar tais adulterantes, padronizou-se técnica em cromatografia de camada delgada comparativa (CCDC) em placa de silica gel para anorexígenos e coadjuvantes do emagrecimento (femproporex, mazindol, anfepramona, fenfluramina, hidroclortiazida, furosemida, fluoxetina, benzocaína, diazepam, midazolan, flurazepam, bromazepam, nitrazepam, clordiazepóxido, cafeína, fenolftaleína) para testar interferência analítica com fitofármacos (carqueja, clorella, fucus, centella, jurubeba, maracujá, alcachofra, gingeng, guaraná, boldo, alcachofra, espinheira-santa, espirulina, citrin extract, aloína, cáscara sagrada, sene). Valores de Rf e cores desenvolvidas pelos fármacos e fitofármacos foram comparados, repetindo CCDC para mistura de possíveis interferentes. Concluiu-se que a técnica é seletiva, rápida, simples e de baixo custo, podendo ser desenvolvida em qualquer laboratório analítico para a identificação das substâncias químicas sintéticas em formulações de fitoterápicos.
\end{abstract}

PALAVRAS-CHAVE: CCDC; cromatografia; anorexigeno; fitoterápico; cromatografia; padronização

ABSTRACT: There are reports of synthetic substances not mentioned in the composition of slimming phytotherapics freely marketed being they responsible for the apparently miraculous effects of these formulations. These substances act in the Central Nervous System (CNS) with sale and use restrictions. Due the need to identify those adulterants, a comparative thin layer chromatography technique (TLC) was validated for drugs $(1 \mathrm{mg} / \mathrm{mL}$ in methanol solution) used in the treatment of obesity (femproporex, mazindol, diethylpropion, fenfluramine, hydrochlorotiazide, furosemide, fluoxetine, benzocaine, diazepam, midazolam, flurazepam, bromazepam, nitrazepam, clordiazepoxide, caffeine, phenolphthalein). Analytical interference with $20 \mathrm{mg}$ of standard phytodrugs (Cynara scolynmus, Aloe vera, Peumus boldus, Baccaharis sp, Rhamnus purshiana, Centella asiatica, Garcinia sp, Chorella pyrenoidosa, Maytenus ilicifolia, Spirulina maxima, Fucus sp., Paullinia cupana, Pfaffia paniculata, Amorphophallus konjac, Solanum paniculatum, Passiflora alata Cassia angustifolia) extracted with $5 \mathrm{~mL}$ of 
methanol was tested. Analytical technique of TLC was developed in silica gel G60's plate, $0.5 \mathrm{~mm}, 20 \times 20 \mathrm{~cm}$, with mobile phase and sequence of detection reagents for substances of acid/neutral and basic characters. The Rf's values and developed spot colors were considered. The procedure for the "natural products" was performed, whose results were compared to the patterns, with repetitions for the suspicious products. It was concluded that the technique is selective, fast and simple and have low cost. It could be developed at any analytic laboratory for the identification of the synthetic anorexic drugs in phytomedicine composition.

KEYWORDS: TLC; chromatography; anoretic drug, phytotherapics, validation.

\section{INTRODUÇÃO}

A obesidade, no sentido estrito, consiste num aumento das reservas lipídicas armazenadas sob a forma de triglicérides no tecido adiposo (ANDREOLLI et al, 2000). Estima-se que 40 milhões de brasileiros são obesos, o que constitui um problema de saúde pública. Além disto, há uma cobrança social por uma silhueta perfeita, que deixa o indivíduo obeso com a auto-estima baixa e com disposição a fazer de tudo para perder peso (ZACHÉ et al, 2000). Dentre os vários tratamentos disponíveis, está o uso de fármacos sintéticos e produtos naturais.

Relatos na literatura (CFF, 1997; VALLADÃO \& LISBOA, 1992) e através de observações pessoais, percebe-se que a automedicação com "produtos naturais" emagrecedores tem aumentado consideravelmente nos últimos anos, visto que a população, em geral, acredita que estes medicamentos não trarão danos a saúde. Corrobora com esta crença, a existência de propaganda enganosa de algumas indústrias farmacêuticas que visam ao lucro sem se preocupar com a saúde pública. No entanto, conforme demonstrado por pesquisas, muitos produtos vendidos como "naturais" mostram a adição, não declarada nas formulações, de substâncias anorexígenas sintéticas e coadjuvantes (benzodiazepínicos, antidepressivos) (CUNHA et al, 2000; ALMEIDA \& RIBEIRO, 1998; CHASIN et al, 1998; LUCAS et al, 1997; PERLINGEIRO et al, 1997; SANTOS et al, 1997; CHAVES et al, 1994; NAPPO \& CARLINI, 1993; NAPPO, 1992; AURICCHIO et al, 1991; ALMEIDA \& RIBEIRO, 1999) inclusive, em produtos chineses tradicionais (RAY et al, 1999; CHANG et al, 2000).

Dentre os grupos de fármacos antiobesidade comercializados no Brasil (cartáticos/laxantes, hormônios, produtos naturais, anestésicos locais e aminas simpatomiméticas), este último (fármacos anorexígenos sintéticos) estão freqüentemente envolvidas em abuso e, além de serem responsáveis pelos efeitos anorexígenos miraculosos das formulações para emagrecer, possuem restrições e contra-indicações de uso, podendo levar à tolerância, dependência química e síndrome de abstinência (KOROLKOVAS \& BURKHALTER, 1982; HARDMAN \& LIMBIRD, 1996).

Há vários métodos e técnicas analíticas para identificação de substâncias, os quais são escolhidos avaliando desde fatores referentes à substância (propriedades químicas, físicas e biológicas) como também do método (disponibilidade, simplicidade, viabilidade financeira, adequação técnica, dentre outros). Um método muito usado para estudar a adulteração de fitoterápicos emagrecedores por fármacos anorexígenos e coadjuvantes (benzodiazepínicos, diuréticos) é a cromatografia em camada delgada comparativa (CCDC), porque os fármacos estão na ordem de miligramas e por apresentar procedimentos analíticos mais rápidos, robustos, abrangentes, de baixo custo e que vem auxiliando na identificação dos grupos de substâncias supracitados (AURICCHIO et al, 1991).

Sabendo dos efeitos indesejados já descritos que tais adulterantes podem causar, além da necessidade de alertar toda a sociedade sobre os perigos advindos do uso e abuso de substâncias psicoativas nas formulações para emagrecimento, resolveu-se padronizar técnica analítica em CCDC para possibilitar o estudo da incidência de adulterantes sintéticos em produtos fitoterápicos emagrecedores. 


\section{METODOLOGIA}

A análise cromatográfica dos fármacos anorexígenos sintéticos e dos fitoterápicos baseou-se na metodologia clássica de CCDC descrita por MORAES, $1991 \mathrm{com}$ adaptação. Para garantir a performance do método, realizaram-se análises cromatográficas do fármaco sintético, do fitofármaco de referência, da associação de fitofármacos e da associação de fármaco sintético com fitofármacos. Utilizaram-se, como parâmetros de validação, a comparação do $\mathrm{Rf}$ (fator de retenção) e da cor desenvolvida na seqüência de reveladores empregados para a detecção do perfil cromatográfico do fitoterápico e dos padrões de fármacos sintéticos analisados concomitantemente.

Para a análise dos fármacos sintéticos (femproporex, mazindol, anfepramona, fenfluramina, furosemida, fluoxetina, benzocaína, diazepam, midazolam, flurazepam, bromazepam, nitrazepam, clordiazepóxido, cafeína), cedidos gentilmente por indústrias fabricantes com certificado de pureza, submeteu-se $20 \mu \mathrm{L}$ da solução padrão de $1 \mathrm{mg} / \mathrm{mL}$ em metanol à CCDC para fármacos de caráter básico (anorexígenos sintéticos) e de caráter ácido/neutro (benzodiazepínicos), conforme Figura 1. A sibutramina (Plenty®, Medley e Reductil@, Abbott) não foi analisada por estar indisponível no mercado (indústrias e farmácias de manipulação) à época da pesquisa.

\begin{tabular}{|c|c|c|}
\hline $\begin{array}{l}\text { Fármacos sintéticos } \\
\text { 1. mazindol } \\
\text { 2. femproporex } \\
\text { 3. anfepramona } \\
\text { 4. fenfluramina } \\
\text { 5. hidroclortiazida } \\
\text { 6. furosemida } \\
\text { 7. fluoxetina } \\
\text { 8. benzocaína } \\
\text { 9. diazepam } \\
\text { 10. midazolam } \\
\text { 11. } \text { flurazepam } \\
\text { 12. bromazepam } \\
\text { 13. nitrazepam } \\
\text { 14. clordiazepóxido } \\
\text { 15. cafeína }\end{array}$ & $\begin{array}{c}\text { Padrão (*) } \\
\text { (1 mg/ml em Metanol) } \\
\text { ácido/neutro } \\
\text { caráter } \\
\text { Mnotar cores desenvolvidas }\end{array}$ & $\begin{array}{l}\text { Seqüência de Reveladores } \\
\text { Caráter ácido/neutro: } \\
\text { 1. } \quad \text { Luz UV }(\lambda=264 \mathrm{~nm}) \\
\text { 2. } \quad \text { Cloreto férrico } \\
\text { 3. } \quad \text { Calor }\left(105^{\circ} \mathrm{C}\right) \\
\text { 4. } \quad \text { Reativo de Dragendorff } \\
\text { 5. } \quad \text { Reativo Iodoplatinado ácido } \\
\text { Caráter básico: } \\
\text { 1. Luz UV }(\lambda=264 \mathrm{~nm}) \\
\text { 2. Ninidrina/calor }\left(105^{\circ} \mathrm{C}\right) \\
\text { 3. } \text { Reativo de Forrest } \\
\text { 4. Reativo de Iodoplatinado } \\
\text { 5. } \text { Reativo de Dragendorff. }\end{array}$ \\
\hline
\end{tabular}

Figura 1. Condições cromatográficas para análise dos fármacos sintéticos (padrões, à esquerda), incluindo a seqüência de reveladores utilizados na revelação dos fármacos de caráter ácido e básico.

Aos fitoterápicos de referência (FR) adicionaram-se metanol (20 mg do produto com $5 \mathrm{~mL}$ de metanol) para extrair eventuais anorexígenos sintéticos presentes, além de biomarcadores dos mesmos. Após evaporação, adicionou $0,2 \mathrm{~mL}$ de metanol ao resíduo para realização da CCDC tanto para fármaco de caráter ácido/neutro quanto para de caráter básico, conforme a Figura 2. Os produtos de referência utilizados foram cedidos por uma farmácia de manipulação conceituada com ISO 9002 e são eles: alcachofra (Cynara scolynmus), aloe (Aloe vera), boldo (Peumus boldus Mol.), carqueja (Baccaharis sp), cáscara sagrada (Rhamnus purshiana), Centella asiatica, citrin extract (Garcinia sp), clorela (Chorella pyrenoidosa), espinheira santa (Maytenus ilicifolia), espirulina (Spirulina maxima), Fucus sp., guaraná (Paullinia cupana), ginseng falso (Pfaffia paniculata), glucomanan (Amorphophallus konjac), jurubeba (Solanum paniculatum), maracujá (Passiflora alata) e sene (Cassia angustifolia). 




Figura 2. Condições cromatográficas para análise dos fitofármacos de referência (à esquerda), revelados com os mesmos reveladores utilizados para os padrões sintéticos (direita).

Os resultados (Rf e cores desenvolvidas) dos fitoterápicos de referência foram comparados com os dos padrões de anorexígenos sintéticos para verificar possíveis interferências, tais como sobreposição de substâncias com Rfs idênticos ou similares e alteração da cor da mancha, que podem ocorrer na análise dos produtos ditos "naturais".

As placas cromatográficas utilizadas foram confeccionadas em suporte de vidro $20 \times 20 \mathrm{~cm}$, com espessura de $0,5 \mathrm{~mm}$ de sílica gel G60 (Merck). Para tal, preparou-se uma emulsão com $40 \mathrm{~g}$ de sílica, $80 \mathrm{~mL}$ de água destilada e $0,5 \mathrm{~mL}$ de etanol $96^{\circ} \mathrm{GL}$ que foi aplicada sobre 6 placas de vidro. Estas foram secas à temperatura ambiente e, antes do uso, foram ativadas por 1 hora a $110^{\circ} \mathrm{C}$. A corrida cromatográfica foi realizada em cuba cromatográfica DESAGA ${ }^{\circledR}$ e padronizada em $10 \mathrm{~cm}$ de altura.

\section{RESULTADOS E DISCUSSÃO}

Os resultados cromatográficos (Rf e cores desenvolvidas) dos fármacos, tanto para os de caráter ácido/neutro como para os de caráter básico, estão dispostos nas tabelas 1 e 2 . Foram feitas repetições da CCDC para os fármacos sintéticos ( $n=10$, para os de caráter básico e $n=8$, para os de caráter ácido/neutro) 0 que possibilitou a obtenção de Rf mediano e da cor desenvolvida. 
Tabela 01. Rf e cor desenvolvida para os fármacos de fármacos de caráter básico, conforme o revelador empregado (Mediana, $\mathrm{N}=10$ ).

\begin{tabular}{|c|c|c|c|c|c|}
\hline \multirow{2}{*}{ Fármaco } & \multicolumn{5}{|c|}{ Reveladores } \\
\hline & Luz UV & Ninidrina/Calor & R. Forrest & R. lodoplatinato & R. Dragendorff \\
\hline \multirow{2}{*}{ Furosemida } & 0,13 & 0,11 & 0,11 & 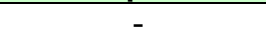 & - \\
\hline & azul & rosa & vermelha & & \\
\hline \multirow{2}{*}{ Fluoxetina } & - & 0,51 & - & 0,51 & 0,51 \\
\hline & & roxa & & branca & branca \\
\hline \multirow{2}{*}{ fenfluramina } & - & 0,58 & - & - & - \\
\hline & & roxa & & & \\
\hline \multirow[b]{2}{*}{ Mazindol } & - & 0,77 & - & - & - \\
\hline & & vermelha & & & \\
\hline \multirow{2}{*}{ anfepramona } & 0,96 & 0,96 & 0,96 & 0,96 & 0,96 \\
\hline & marrom & amarela & laranja & rosa & laranja \\
\hline \multirow{2}{*}{ femproporex } & - & 0,95 & - & - & - \\
\hline & & roxa & & & \\
\hline Benzocaína & $\begin{array}{c}0,96 \\
\text { amarela }\end{array}$ & $\begin{array}{c}0,96 \\
\text { vermelha }\end{array}$ & - & - & - \\
\hline
\end{tabular}

Legenda: $(-)$ = não detectado.

Tabela 02. Rf e cor desenvolvida para os fármacos de fármacos de caráter ácido/neutro, conforme o revelador empregado (Mediana, $\mathrm{N}=8$ ).

\begin{tabular}{|c|c|c|c|c|c|}
\hline \multirow{2}{*}{ Fármaco } & \multicolumn{5}{|c|}{ REVELADORES } \\
\hline & Luz U.V. & Cloreto férrico & Calor & R. Dragendorff & R. lodoplatinato ácido \\
\hline \multirow{2}{*}{ bromazepam } & 0,20 & - & - & 0,20 & 0,20 \\
\hline & azul & & & marrom & roxa \\
\hline \multirow{2}{*}{ cafeína } & & - & - & 0,45 & 0,45 \\
\hline & - & & & marrom & roxa \\
\hline \multirow{2}{*}{ clordiazepóxido } & - & - & - & 0,14 & 0,14 \\
\hline & & & & marrom & roxa \\
\hline \multirow{2}{*}{ diazepam } & 0,75 & & - & 0,75 & 0,75 \\
\hline & incolor & & & marrom & roxa \\
\hline \multirow{2}{*}{ furosemida } & - & 0,04 & 0,04 & 0,04 & 0,04 \\
\hline & & castanha & Cinza & cinza & cinza \\
\hline flurazepam & & & & $\begin{array}{c}0,10 \\
\text { marrom }\end{array}$ & $\begin{array}{l}0,10 \\
\text { marrom }\end{array}$ \\
\hline
\end{tabular}




\begin{tabular}{l|c|c|c|c|c}
\hline \multirow{2}{*}{ midazolam } & & - & - & 0,25 & 0,25 \\
& - & & & marrom & roxa \\
\hline \multirow{2}{*}{ nitrazepam } & 0,54 & - & - & 0,54 & 0,54 \\
& marrom & & & marrom & Roxa \\
\hline
\end{tabular}

Legenda: (-) = não detectado.

Apesar de alguns Rfs iguais (anfepramona e benzocaína) ou bem próximos (anfepramona, benzocaína e mazindol), a identificação destas substâncias foi possível devido à diferença nas cores da mancha apresentada em cada revelador e/ou na seqüência de reveladores utilizados.

Para fazer a discriminação de resultados foram analisados os Rfs e a coloração das manchas desenvolvidas nos respectivos reveladores, devendo haver coincidência de resultados em toda a seqüência de reveladores. Por isso, não se fez comparação específica entre produtos naturais e padrões sintéticos que apresentaram discrepância de resultados (seletividade existente), ou que desenvolveram manchas de cores diferentes nos mesmos reveladores, mesmo que obtivessem o mesmo Rf.

Durante as comparações de resultados, consideraram-se apenas os fitoterápicos de referência que apresentaram resultados próximos aos dos padrões de fármacos sintéticos. Nestes casos, foram feitas novas aplicações visando à identificação precisa. Este procedimento de repetição da análise não é adequadamente descrito por outros trabalhos que utilizaram a cromatografia em camada delgada (SANTOS et al, 1997; AURICCHIO et al, 1991). Apesar de alguns fitofármacos (carqueja, guaraná, espinheira santa, espirulina, aloína, cáscara sagrada) terem apresentados Rfs próximos aos de fármacos sintéticos (mazindol, anfepramona, furosemida, fluoxetina, midazolam, nitrazepam e bromazepam) não ocorreu interferência analítica apreciável, já que as manchas dos fitoterápicos não possuem comportamento igual a dos padrões sintéticos frente à seqüência de reveladores.

Neste trabalho, a separação e identificação de biomarcadores dos produtos fitoterápicos não foi almejado. As condições cromatográficas tinham que ser adequadas apenas para a separação e identificação dos contaminantes anorexígenos sintéticos.

Por fim, primou-se por escolher técnica simples, rápida e de baixo custo que possa ser utilizada em outros laboratórios com o objetivo de auxiliar na triagem de adulteração de fitoterápicos emagrecedores, visto que a maioria dos outros trabalhos utilizou técnicas mais dispendiosas como espectrofotometria UV/Vis (CHAVES et al, 1994), cromatografia líquida de alta eficiência (ALMEIDA \& RIBEIRO, 1999), cromatografia gasosa (LUCAS et al, 1997; CHANG et al, 2000) e eletroforese capilar (RAY et al, 1999).

A adaptação da técnica analítica para obtenção de extratos de padrões de fitoterápicos e de "produtos naturais" com uso do metanol mostrou-se adequada, visto que o objetivo era analisar a interferência de substâncias presentes nestes produtos com os padrões de fármacos sintéticos solúveis em metanol.

\section{CONCLUSÃO}

A técnica em CCDC mostrou-se reprodutível, robusta, abrangente, precisa e de fácil execução, sendo adequada para procedimentos de triagem de adulteração de produtos naturais por fármacos sintéticos.

A interferência dos fitofármacos na análise dos fármacos sintéticos é desprezível e, uma vez suspeita, deve-se buscar confirmação por outras técnicas e/ou outros métodos de análise - incluindo cromatografia líquida de alta eficiência (CLAE) e cromatografia gasosa (CG). 


\section{REFERÊNCIAS BIBLIOGRÁFICAS}

ANDREOLLI, R.S., CASTRO, R.C., MENDES, R.A. Obesidade. Racine, São Paulo, v.57, p. 38-47, 2000.

ZACHÉ, J., CASTELLAN, L., BOCK, L. Medidas extremas. ISTOÉ. São Paulo : Três, ed. v. 1621, p. 116-122, 25 out. 2000.

CFF - Conselho Federal de Farmácia. A selva dos fitoterápicos. Brasília. Infarma. v. 6, n. 3/4, p. 17-19, 1997.

VALLADÃO, M. L. F, LISBOA, S. M. Cápsulas para emagrecer: uma abordagem legal. Infarma, Brasília, v. 1, n. 1, p. 17-19, 1992.

CUNHA, L. C.; AZEREDO, F. S.; GUIMARÃES, R. I; PAULA, J. R.. Padronização de técnica analítica para análise cromatográfica de fármacos anorexígenos fitoterápicos utilizados no emagrecimento. In: XI CONGRESSO LATINOAMERICANO DE TOXICOLOGIA - ALATOX 2000, 2000, Campinas-SP. Revista Brasileira de Toxicologia. São Paulo-SP: Sociedade Brsileira de Toxicologia, v. 13, p. 104-104, 2000.

ALMEIDA, A.E., RIBEIRO, M.L. Agentes anorexígenos: aspectos químico-farmacológicos e perspectivas. Revista Brasileira de Farmácia, Rio de Janeiro, v. 79, n. 3/4, p. 62-64, 1998.

CHASIN, A.A.M.; NASCIMENTO, E.S.; RIBEIRO-NETO, L.M., et al. Validação de métodos em análises toxicológicas: uma abordagem geral. Revista Brasileira de Toxicologia. São Paulo, v. 11, n. 1, p. 1-6, jul./1998.

LUCAS, T.S., KOLBERG, K., THIESEN, F.V. Análise de Medicamentos não empregados sob prescrição média por cromatografia a gás com coluna megabore. Revista Brasileira de Toxicologia. São Paulo : SBTOX, v. 10, n. 2, p. 104, 1997 (supl.).

PERLINGEIRO, R., BINCOLETTO, $C$ et al. Determinação de anorexígenos, benzodiazepínicos e diuréticos em formulações comercializadas como "naturais". Revista Brasileira de Toxicologia. São Paulo : SBTOX, v. 10, n. 2, p. 98, 1997 (supl.).

SANTOS, P.D., GALVÃO, J.F., LUCAS, A.C.S. Identificação de compostos anfetamínicos em preparações para emagrecer, à base de plantas, produzidas e/ou comercializadas na cidade de Manaus-AM. Revista Brasileira de Toxicologia., São Paulo : SBTOX, v.10, n.2, p.91, 1997 (supl.).

CHAVES, M.A., KATUKA, A.S., TRUJILLO, L.M. Dietilpropiona, femproporex, diazepam e fenolftaleína: determinação em formulações para emagrecimento. Revista do Instituto Adolfo Lutz. Rio de Janeiro, v. 54, n. 1 , p. 36-43, 1994

NAPPO, S.A.; CARLINI, E.A. Revisão: medicamentos anorexígenos. Infarma, Brasília: CFF, v. 2, p. 6-9, set./out. 1993.

NAPPO, S.A. Consumo de anorexígenos tipo-anfetaminas (dietilpropiona, fenproporex, mazindol) e de fenfluramina no Brasil: prejuízo ou benefício para a Saúde. Jornal Brasileiro de Psiquiatria, São Paulo : ECN, v. 41, n. 8, p. 417-421, set. 1992.

AURICCHIO, M.T, ATISTIC, M.A., MARKMAN, B.E.O. Detecção de anorexígenos e benzodiazepínicos em formulações "naturais" empregadas em regimes de emagrecimento. Revista Instituto Adolfo Lutz, Rio de Janeiro, v. 51, n. 1/2, p. 105-110, 1991.

ALMEIDA, A.E., RIBEIRO, M.L. High-performance liquid chromatographic determination of amfepramone hydrochloride, mazindol, and diazepam in tablets. Journal of Liquid Chromatography and related Technologies., v.22, n.11, p. 1759-69, 1999.

RAY, K.Y., SHIUN, C.Y. et al. Analysis and confirmation of synthetic anorexics in adulterated traditional Chinese medicines by high-performance capillary eletroforesis. Journal of Chromatography-A. v. 848, n. 1-2, p. 5375432, 1999. 
CHANG, T.M., JER, T.M. et al. GC/MS analysis on anorexics adulterated in traditional Chinese medicines. Journal of Food and Drug Analysis. v. 8, n. 4, p. 315-30, 2000.

KOROLKOVAS, A., BURKHALTER, J.H. Química Farmacêutica. Rio de Janeiro: Guanabara Dois, 1982.

HARDMAN, J.G. \& LIMBIRD, L.E. Goodman \& Gilman's The Phamacological Basis of Therapeutics, $9^{\text {th }}$ ed., p. 1905, McGraw Hill, New York-USA, 1996.

MORAES, E.C.F.; FERNICOLA, N.A. Manual de Toxicologia Analítica. São Paulo : Roca, 1991. p. 229. 